\title{
Environmental control of defensive reactions to a cat
}

\author{
ROBERT J. BLANCHARD, KENNETH K. FUKUNAGA, and D. CAROLINE BLANCHARD \\ University of Hawaï, Honolulu, Hawaï 96822
}

\begin{abstract}
Brief familiarization with a strange environment changes the defensive reactions of rats confronted by a cat in that environment, from flight to freezing. Thus flight appears to be a dominant initial reaction to threat stimuli unless the threatened animal is familiar with the lack of availability of escape from the threat situation. Further, as attempts at flight familiarize the subject with the inescapable situation, flight becomes less dominant and freezing emerges as the major defensive reaction. This interpretation supports an elicitation view of flight and freezing, in which features of environmental stimuli, as well as the threat stimuli themselves, determine the topography of elicited defensive reactions.
\end{abstract}

The defensive reactions of the domesticated rat to nonconspecific threat stimuli typically consist of either flight (Blanchard \& Blanchard, 1969b) or freezing (Blanchard \& Blanchard, 1969a). Logically, it is clear that either of these reactions may enhance the rat's prospects of avoiding or escaping from the threat source, under suitable circumstances. Specifically, if a means of escape is available, then flight is usually the more beneficial response, while freezing is advantageous when escape is impossible.

Two general mechanisms to account for the correspondence of defensive reactions, and features of the threat situation, involve either the effects of specific rewards and punishments or some type of elicitation model. Thus, it has been suggested (Bolles, 1970) that individual defensive responses drop out when they are punished, leaving appropriate (i.e., successful) behaviors as the dominant response to threat in a particular situation. In contrast, an elicitation model (Blanchard \& Blanchard, 1969b) would suggest that the specific typology of defensive behavior is determined directly by biologically important features of the threat source and situation, presumably on the basis of differential gene survival for animals making these specific responses under such circumstances. Bolles' (1975) "expectancy" model is a related notion, in that it provides for different defensive behaviors, without punishment effects. Clearly, then, a choice between these two views depends on whether features of threat situations or stimuli can influence the form of defensive behavior when no defensive responses made are followed by punishment.

The present study, therefore, presents a comparison of freezing and flight responses to an unconditioned threat stimulus (cat) for rats given a short exploration period in an inescapable chamber (delayed threat group). Rats display strong defensive reactions to a cat, even when rat-cat contact is not allowed and in the absence of

Requests for reprints should be sent to Robert J. Blanchard, University of Hawaii, Department of Psychology, 2430 Campus Road, Honolulu, Hawaii 96822. previous experience with a cat (Blanchard \& Blanchard, 1971). This factor makes it possible to examine the effects of situational variables on the topography of defensive reactions, in a task not involving the use of painful stimulation.

\section{METHOD}

\section{Subjects}

All subjects were naive male albino rats, 116-179 days of age, from the colony maintained by the University of Hawaii Department of Psychology. None of the rats had been handled or exposed to a cat at any time before the experiment. All subjects were housed singly for at least 3 weeks prior to the experimental procedures. The stimulus cat was a $2.3-\mathrm{kg}$ gray Tiger cat obtained from the Pacific Biomedical Research Center Colony of the University of Hawaii.

\section{Apparatus}

The apparatus was a $90 \times 90 \times 90 \mathrm{~cm}$ plywood box with a clear Plexiglas front to permit observation. The box was divided into a $60 \times 90 \times 90 \mathrm{~cm}$ rat compartment and a $30 \times 90 \times 90 \mathrm{~cm}$ cat compartment by a wire mesh screen running from the front to the back. Lines on the floor of the rat compartment divided it into six $30 \times 30 \mathrm{~cm}$ sections to facilitate measurement of activity. An 8-W fluorescent fixture providing 20-22 fc was mounted on the center of the roof of the rat compartment. A loudspeaker mounted outside the cat chamber provided 65-dB SPL speech noise from a Grason-Stadler white noise generator. Behaviors of the rat and the stimulus cat were observed from an adjacent room through a one-way vision mirror.

\section{Procedure}

Eight subjects were randomly assigned to each of three groups. Rats of the immediate-cat group were introduced into the rat compartment at the same time as the cat was placed in the cat compartment, then observed for $5 \mathrm{~min}$. The delayed-cat group was introduced into the rat compartment $2 \mathrm{~min}$ before the introduction of the cat and the initiation of a 5-min session. These rats were briefly picked up and replaced in the apparatus as the cat was introduced, to provide a control for the handling given at the same time (with reference to introduction of the cat) for the immediate-cat group. The no-cat control rats were left in the apparatus for the entire 7-min session without the cat.

Response measures included movement time and the line crossings during each minute for the rats. Videotapes were made of the rats' behavior immediately upon the introduction of the 


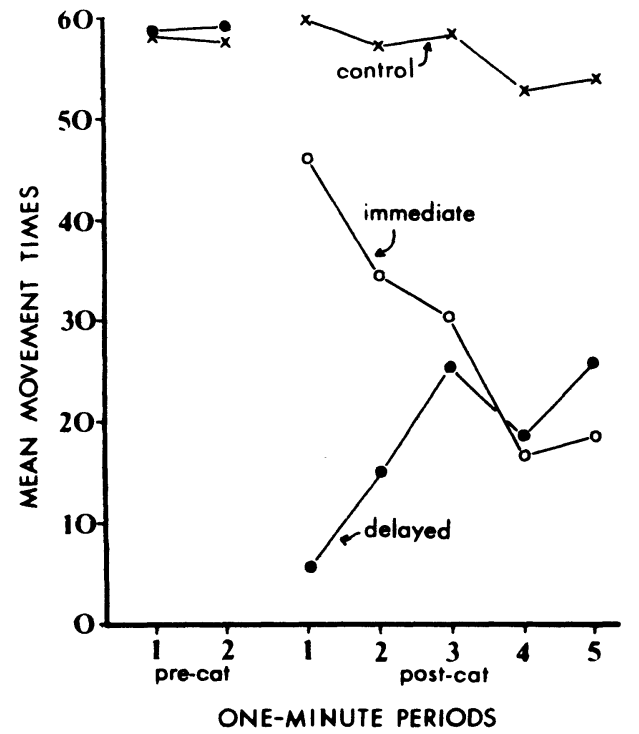

Figure 1. Mean movement times for each 2 min of the test periods for rats confronted with the cat immediately after placement in the test situation, or after a delay, and for no-cat controls.

cat, so that the initial defensive reactions elicited by the cat could be reexamined.

\section{RESULTS AND DISCUSSION}

Mean movement times for each minute of the test periods are presented in Figure 1. As may be seen, movement times for the delayed-cat group, before the cat was introduced, were not different from those of control (no-cat) rats $[t(14)=.68, p>.05]$. However, when the cat was placed in the apparatus, an immediate suppression of activity was seen [ $\mathrm{t}(14)=11.07, \mathrm{p}<.001$, for comparison of control- and delayed-cat groups during the first two post-cat minutes]. The rats placed into the apparatus at the same time as the cat also showed less activity during this period than did the controls $[t(14)=4.16, p<.001]$. However, they were reliably more active in the first $2 \mathrm{~min}$ with the cat than the rats which had been allowed to explore the test situation before the cat was introduced [ $\mathrm{t}(14)=5.29, \mathrm{p}<.001]$.

This clear initial difference in freezing between immediate and delayed groups strongly suggests that familiarity with an inescapable chamber leads to freezing, rather than flight, as the dominant reaction to threat in that situation. This difference occurs in the initial period of response to threat, suggesting that continued or additional punishment of flight is not necessary for the appearance of strong freezing reactions.

Figure 1 also indicates that the initial difference in suppression of activity for the delayed-and immediatecat groups disappeared with increasing time after introduction of the cat. During the last $3 \mathrm{~min}$ of the 5 -min cat period, no activity differences were obtained for these groups $[t(14)=.25]$. Since the first $2 \mathrm{~min}$ in the apparatus for the immediate-cat group should have served to familiarize these rats with the inescapable chamber, this finding is also in agreement with the notion that familiarity with an inescapable chamber reduces flight and potentiates freezing.

Initial (immediate) reactions of rats to the cat, and line crossings, are presented in Table 1 . Line crossings for the three groups were in good agreement with the movement time data: The delayed-cat group showed reliable suppression of line crossings in comparison with controls during Minutes 3 and 4 of testing, i.e., just after the cat was introduced for the delayed-cat group $[\mathrm{t}(14)=2.61, \mathrm{p}<.05]$. Also, the immediate-cat group showed less suppression of line crossing than did the delayed-cat group $[\mathrm{t}(14)=3.24, \mathrm{p}<.01]$ during the first $2 \mathrm{~min}$ after introduction of the cat. Finally, the line crossings data agree with those on movement times in showing a reduction in activity for the immediate-cat group after $2 \mathrm{~min}$ in the cat box. Thus, during the last 3 min of confrontation with the cat, the delayed-cat and immediate-cat groups were not different in line crossings $[t(14)=1.40]$. Some differences between the movement time and line crossings data did appear, however. Line crossings before introduction of the cat were higher for the delayed-cat group than for controls $[t(14)=2.64, p<.05]$. Since the groups were randomly constituted and had received identical treatment at this point, such a difference is apparently due to chance variation. In any event, the obtained difference is opposite to differences occurring after the cat was introduced, so there is no problem of confounding.

The initial reactions of the rats to the cat also agree with the movement time and line crossings results, supporting an hypothesis that familiarity with an inescapable situation potentiates freezing: Five of the eight subjects of the delayed-cat group showed freezing as the initial response to the cat, while none of the eight immediate-cat subjects froze. A Fisher exact probability test (Siegel, 1956) indicated that this difference was reliable $(\mathrm{p}=.05)$.

Table 1

Line Crossings and Initial Reactions to a Cat for Rats Confronted with the Cat Immediately or After Delay and for No-Cat Controls

\begin{tabular}{lccc}
\hline & $\begin{array}{c}\text { No-Cat } \\
\text { Control }\end{array}$ & $\begin{array}{c}\text { Immediate } \\
\text { Cat }\end{array}$ & $\begin{array}{c}\text { Delayed } \\
\text { Cat }\end{array}$ \\
\hline Minutes 1-2, pre-cat & \multicolumn{3}{c}{ Line Crossings* } \\
Minutes 1-2, post-cat & 5.13 & & 13.06 \\
Minutes 3-5, post-cat & 5.45 & 6.37 & 1.75 \\
& Initial Reaction to the Cat \\
Percent approach & \multicolumn{3}{c}{5.29} \\
Percent withdraw & & 50.00 & 12.5 \\
Percent freeze & & 50.00 & 25.0 \\
& & 0.0 & 62.5 \\
\hline
\end{tabular}

* Line crossings figures represent mean per minute values. 
Thus, the present results clearly support a rather cognitive model for the determination of whether flight or freezing will be the major mode of defensive responding to a threat stimulus. Since no contact of the rat and cat was permitted, physical punishment of responses was not a factor in this task. Also, since neither flight nor freezing resulted in the removal of the cat, "punishment" as the continuing presence of the threatening stimulus could not have differentially affected the persistence of flight vs. freezing.

\section{REFERENCES}

Blanchard, R. J., \& Blanchard, D. C. Crouching as an index of fear. Journal of Comparative and Physiological Psychology, 1969, 67, 370-375. (a)
Blanchard, R. J., \& Blanchard, D. C. Passive and active reactions to fear-eliciting stimuli. Journal of Comparative and Physiological Psychology, 1969, 68, 129-135. (b)

Blanchard, R. J., \& BlanchaRd, D. C. Defensive reactions in the albino rat. Learning and Motivation, 1971, 2, 351-362.

Bolles, R. C. Species-specific defense reactions and avoidance learning. Psychological Review, 1970, 71, 32-48.

Bolles, R. C. Learning theory. New York: Holt, Rinehart and Winston, 1975.

SIEGEL, S. Nonparametric statistics for the behavior sciences. New York: McGraw-Hill, 1956.

(Received for publication May 3, 1976.) 